\title{
More than One Antigen Contributes to the Immunogenicity of Neisseria gonorrhoeae in the Guinea Pig Chamber Model
}

\author{
By N. J. PARSONS, C. W. PENN, D. R. VEALE AND H. SMITH \\ Department of Microbiology, University of Birmingham, P.O. Box 363, Birmingham B15 2 TT
}

(Received 22 November 1978)

Gonococci adapted to growth in guinea pig chambers [strain BS4 (agar)] were predominantly smooth organisms and produced a type-specific antigen. A vaccine prepared by treating these gonococci with formalin, protected guinea pig chambers against homologous challenge in contrast to a similarly treated laboratory strain (BSDH) which had been selected in vitro from the same parent strain and which did not produce the type-specific antigen. Surface washes of BS4 (agar) contained the type-specific antigen but attempts to immunize guinea pigs with complexes of rabbit antibody with this antigen excised from gels failed. However, good immunity could be produced by combining such complexes with formalin-killed rough gonococci (strain BS4R), lacking the type-specific antigen, which were found in some chambers of challenged guinea pigs that had been immunized with the complexes. Hence, at least two antigens - one the type-specific antigen and the other(s) possessed by both BS4 (agar) and BS4R - are needed for immunogenicity. Surface washes of BS4 (agar) and BS4R contained three antigens, distinct from the type-specific antigen, which might complement it in producing immunity. Similar antigens were present in surface washes of five fresh isolates from human urethral pus, but only a few organisms from these isolates possessed antigens similar to the type-specific antigen. The variability of gonococci in antigenicity, immunogenicity and probably virulence is discussed.

\section{INTRODUCTION}

One animal model developed to demonstrate the immunogenicity of Neisseria gonorrhoeae and its products is the infection of perforated plastic chambers implanted subcutaneously into guinea pigs: strain-specific immunity to chamber infection has resulted from either spontaneous eradication of a previous chamber infection or repeated subcutaneous inoculation of large quantities of killed gonococci (Arko, 1974; Scales \& Kraus, 1974; Turner \& Novotny, 1976; Chandler et al., 1976; Wong et al., 1976; Penn et al., 1977a; Arko \& Wong, 1977; Novotny et al., 1978). The nature of the immunizing antigens is now under investigation. Active immunity studies in the guinea pig model (Buchanan et al., 1977) indicate that strain-specific pili antigens are not strongly protective. However, significant protection was achieved in guinea pigs with isolated outer membrane complex which, although purified, was a mixture of antigenic components (Buchanan \& Arko, 1977; Buchanan et al., 1977). In other animal models, lipopolysaccharide (Diena et al., 1978) and a cytoplasmic component (Kwapinski et al., 1976) have been implicated in immunity, and Robbins (1977) has pointed out that immunity to $N$. gonorrhoeae may require the combined effect of a number of antigens.

Our objective was to ascertain whether gonococci adapted to growth in vivo in guinea pig chambers did produce immunizing antigens differing in quality or quantity from those 
produced by organisms grown in vitro. Gonococci adapted to growth in vivo in guinea pig chambers differ from those grown in vitro in a number of properties including the possession of at least one surface antigen not detected in surface washes of the parent in vitro-grown organisms (Penn et al., 1976). However, neither chamber-grown organisms nor their extracellular products present in chamber fluid were particularly immunogenic since small doses of formalin-killed gonococci obtained directly from chambers and filtered fluid from the infected chambers did not immunize guinea pigs against chamber infection (Penn et al., $1977 a$ ). Nevertheless, surface washes of three immunotypes of $N$. gonorrhoeae adapted to growth in guinea pig chambers and grown once on agar revealed, in two-dimensional immunoelectrophoresis against rabbit antisera, evidence for an antigen of similar electrophoretic behaviour for each immunotype but without cross-reactivity between them (Penn et al., 1978). The type-specific antigens might therefore contribute to immunity; they were not found in surface washes of the parent strain grown in vitro but were possessed by some colonies within the primary or secondary subculture of human gonorrhoeal pus. The above results together with a report (Arko et al., 1976) that killed mouse chamber-adapted gonococci grown once in vitro were more immunogenic than the corresponding parent strain prompted the investigations described here. First, the immunogenicity of gonococci adapted to growth in guinea pig chambers and then grown once in vitro was compared with that of the parent strain grown in vitro. Secondly, the surface wash of the in vivo-adapted gonococci which contained the type-specific antigen was tested for immunogenicity. Thirdly, the precipitin line formed in gels by the type-specific antigen and rabbit antibody was excised and tested for immunogenicity; this procedure led to further experiments suggesting that more than one antigen is involved in producing immunity to infection.

\section{METHODS}

Gonococcal strains. The parent strain BS, the strain selected from BS in vitro with a 'double highlight' colony morphology [BSDH], and the strain derived from BS by four passages through guinca pig chambers and used directly [BS4] or after one subculture on agar [BS4 (agar)] were described by Veale et al. (1975) and Penn et al. $(1977 a, b)$. Surface washes of BS4 (agar) but not those of BSDH organisms contained the type-specific antigen described previously (Penn et al., 1978). BS4 (agar) organisms formed smooth suspensions in saline (hereafter referred to as smooth) but when plated on agar some clones (6/41 examined) were found which formed rough suspensions in saline (hereafter referred to as rough) and whose surface washes lacked the type-specific antigen. One of these rough clones (clone 3) was used for adsorption of antiserum as described below.

Culture media. The media used for growth, to prepare suspensions and to perform viable counts of the gonococcus were as previously described (Veale et al., 1975; Penn et al., 1976).

Preparation of gonococci for immunization. Strains BS4 and BSDH were grown on a clear agar medium (AG agar) (Veale et al., 1975; Penn et al., 1976) at $37^{\circ} \mathrm{C}$ for 20 to $24 \mathrm{~h}$. The growth was harvested into Trypticase Soy Broth (TSB; BBL) (Penn et al., 1976) and the count was adjusted to $10^{\circ}$ colony-forming units (c.f.u.) gonococci $\mathrm{ml}^{-1}$. The organisms were killed by treatment with $0.25 \%(\mathrm{v} / \mathrm{v})$ neutral formalin at $4{ }^{\circ} \mathrm{C}$ overnight and checked for sterility by plating $0.1 \mathrm{ml}$ suspension on haemoglobin agar (Penn et al., 1976).

Surface wash of strain BS4 (agar) for immunization. Suspensions containing $10^{9}$ and $10^{10}$ c.f.u. BS4 (agar) $\mathrm{ml}^{-1}$ in TSB were vortex mixed for $1 \mathrm{~min}$ at room temp. with $3 \mathrm{~mm}$ diam. glass beads. After centrifugation at $3000 \mathrm{~g}$ and $4{ }^{\circ} \mathrm{C}$ for $10 \mathrm{~min}$, the supernatant fluid was removed, treated with $0 \cdot 25 \%(\mathrm{v} / \mathrm{v})$ neutral formalin as already described and tested for sterility.

Immunization of guinea pigs with killed gonococci or their products, and subsequent challenge. This was done as described by Penn et al. (1977a) except that doses of $10^{7}, 10^{8}$ and $10^{9}$ killed c.f.u. or surface washes equivalent to $10^{7}, 10^{8}, 10^{9}$ and $10^{10}$ c.f.u. were injected on five occasions at weekly intervals, and BS4 organisms (approx. 1000 c.f.u.) were used for challenge of the chambers. Usually each guinea pig had two chambers which were regarded as immune if viable gonococci could not be detected in the chamber fluid (i.e. $<50$ c.f.u. $\mathrm{ml}^{-1}$ ) $5 \mathrm{~d}$ after challenge.

Surface washes for antigenic analysis, and rabbit antisera for two-dimensional immunoelectrophoresis. These were prepared as described by Penn et al. (1978). Antiserum was adsorbed with a suspension of $10^{11} \mathrm{c} . \mathrm{f} . \mathrm{u} . \mathrm{ml}^{-1}$ for $5 \mathrm{~min}$ at room temp. followed by centrifugation at $3000 \mathrm{~g}$ and room temp. for $10 \mathrm{~min}$. After repeating the process twice, a final adsorption with $10^{11}$ c.f.u. $\mathrm{ml}^{-1}$ was done at $4{ }^{\circ} \mathrm{C}$ overnight. 
Immunization of guinea pigs with type-specific antigen/antibody complex excised from two-dimensional immunoelectrophoresis gels. A surface wash of BS4 (agar) organisms was subjected to electrophoresis in two dimensions using a rabbit antiserum prepared against BS3 (agar) organisms [i.e. organisms passaged three times in a chamber and grown once on agar (Penn et al., 1976)] which had been adsorbed with a suspension of the rough, antigen-deficient clone (see above). The precipitin lines, corresponding to the type-specific antigen, from 32 gels were excised with a scalpel, suspended in $1 \mathrm{ml}$ phosphate-buffered saline $\mathrm{pH} 7 \cdot 4$ (PBS) (Penn et al., 1976) and sonicated at $40 \mathrm{~W}$ for 2 min (Rapidis 180; Ultrasonics Ltd, Shipley, Yorks.). The resulting suspension was injected subcutaneously three times (totalling the equivalent of the lines from 6 to 9 gels per animal) at $14 \mathrm{~d}$ intervals into guinea pigs implanted with two chambers. The first two doses were mixed with an equal quantity of Freund's complete adjuvant. Control animals received equivalent quantities of PBS in Freund's complete adjuvant. One to two weeks after the final injection the chambers were challenged with BS4 organisms and the presence of immunity was assessed as already described.

\section{RESULTS}

Immunogenicity of killed in vivo-adapted gonococci [BS4 (agar)] and of the related laboratory maintained strain [BSDH]

A course of five injections of $10^{8}$ killed c.f.u. BS4 (agar) produced a significant degree of immunity against chamber challenge with BS4, whereas no immunity was detected after a similar series of injections of $10^{9}$ c.f.u. BSDH. That this difference in response reflects the absence in the surface wash of BSDH organisms of the type-specific antigen present in the surface wash of BS4 (agar) organisms is suggested by the latter wash exhibiting a small but significant immunizing activity for guinea pigs (Table 1).

\section{Protection of guinea pigs with type-specific antigen/antibody complex from surface washes of} BS4 (agar) organisms

After vaccination with the antigen/antibody complex, antibody to the type-specific antigen was detected by two-dimensional immunoelectrophoresis in the sera of $2 / 3$ guinea pigs inoculated. However, no chambers were immune to challenge with BS4 after such vaccination. Over the first few days following challenge, the numbers of gonococci in the chambers of the animals receiving the antigen/antibody complex were lower $\left(10^{2}\right.$ to $10^{4} \mathrm{c}$.f.u. $\mathrm{ml}^{-1}$ in chamber fluid) than those $\left(0.5 \times 10^{6}\right.$ to $5 \times 10^{6}$ c.f.u. $\mathrm{ml}^{-1}$ in chamber fluid) in the chambers of control animals. The organisms recovered from the chambers on the two guinea pigs whose serum contained antibody to the type-specific antigen formed a rough suspension in saline and gave washes in which the type-specific antigen could not be detected by two-dimensional immunoelectrophoresis. A clone of such organisms from one chamber was designated BS4R. In contrast, the organisms recovered from the chambers on the nonresponding guinea pig formed a smooth suspension in saline and gave washes possessing the type-specific antigen.

Solid immunity conferred by vaccinating with a mixture of the type-specific antigen/antibody complex from BS4 (agar) organisms and killed organisms of the rough strain BS4R

The results in Table 2 confirm that the type-specific antigen/antibody complex when given alone was usually unable to immunize against chamber infection. Only $2 / 18$ chambers in the two experiments were immune to challenge, but again the numbers of c.f.u. in the chambers soon after challenge were lower than those in the controls, and from 11/16 nonimmune chambers rough organisms lacking the type-specific antigen in surface washes were isolated. However, the correlation between animals responding serologically to the antigen/ antibody complex and the recovery of rough organisms was not complete; 4/5 animals examined responded to the complex, yet $3 / 8$ chambers on these four animals contained smooth organisms which produced the type-specific antigen. The striking result was that killed BS4R organisms, given at doses less than those which by themselves did not immunize, produced good immunity when combined with the type-specific antigen/antibody complex, 
Table 1. Immunogenicity for guinea pigs of killed in vivo-adapted gonococci [BS4 (agar)], their surface washes and related laboratory maintained gonococci [BSDH]

Summary of results from three experiments.

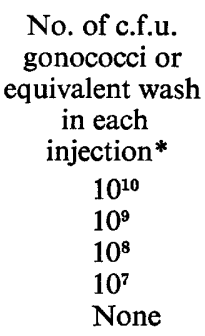

\begin{tabular}{|c|c|c|c|}
\hline \multicolumn{4}{|c|}{$\frac{\text { No. of chambers found immune } \dagger}{\text { No. of chambers challenged }}$} \\
\hline \multicolumn{3}{|c|}{ After vaccination with: } & \multirow[b]{2}{*}{ Controls } \\
\hline BS4 (agar) & BSDH & $\begin{array}{c}\text { Wash of } \\
\text { BS4 (agar) }\end{array}$ & \\
\hline ND & ND & $3 / 6$ & \\
\hline $9 / 10$ & $0 / 10$ & $2 / 9$ & \\
\hline $5 / 7$ & $0 / 9$ & $1 / 8$ & \\
\hline \multirow[t]{2}{*}{$3 / 8$} & $0 / 2$ & $1 / 8$ & \\
\hline & & & $0 / 8$ \\
\hline
\end{tabular}

ND, Not determined.

* Five injections, given at weekly intervals.

$\dagger<50$ c.f.u. gonococci $\mathrm{ml}^{-1}$ in chamber fluid $5 \mathrm{~d}$ after challenge with 1000 c.f.u. BS4; control chambers contained $0.5 \times 10^{6}$ to $5 \times 10^{6}$ c.f.u. $\mathrm{ml}^{-1}$.

i.e. the complex exerted a synergistic effect. Hence, at least two components appeared to be necessary to evoke immunity - the type-specific antigen and an antigen or antigens formed by strain BS4R.

The simplest explanation for the immunizing activity of the rough strain BS4R at high doses $\left(10^{9}\right.$; Table 2$)$ was that such suspensions contained some smooth organisms having the type-specific antigen. This proved to be so, for examination of clones derived from 57 colonies of BS4R revealed four that formed smooth suspensions, and the type-specific antigen was detected in surface washes of two of these clones. Thus, $10^{9}$ c.f.u. BS4R contained about $4 \times 10^{7}(4 \%)$ c.f.u. forming the type-specific antigen and immunized $6 / 10$ chambers (Table 2), a similar proportion to those immunized by injections of $10^{7}$ to $10^{8}$ c.f.u. BS4 (agar) each of which contained about $85 \%$ smooth and $15 \%$ rough organisms (Table 1 ).

The antigens present in surface washes of strains $\mathrm{BS} 4$ (agar), $\mathrm{BS} 4 \mathrm{R}$ and $\mathrm{BSDH}$ as revealed by two-dimensional immunoelectrophoresis

Figure 1 shows the results of two-dimensional immunoelectrophoresis of surface washes of strains BS4 (agar), BS4R and BSDH using rabbit antiserum prepared against BS3 (agar) with or without adsorption with BS4R. With unadsorbed antiserum, the surface wash of BS4 (agar) produced at least six precipitin lines of which at least four were not seen with the surface wash of BSDH. The four most prominent precipitin lines were designated: antigen $a$, the type-specific antigen formerly described (Penn et al., 1978); antigen $b$, a similar but fainter line; antigen $c$, a low peak above the origin well; and antigen $d$, a low peak migrating further in both dimensions than the other antigens (Fig. 1, diagram). The surface wash of BS4R produced precipitin lines corresponding to antigens $b, c$ and $d$ but not $a$. The lack of antigen $a$ and the presence of antigens $b, c$ and $d$ in BS4R was confirmed by work with the adsorbed serum; only the surface wash of BS4 (agar) showed a line that corresponded with antigen $a$ (Fig. 1).

Preliminary investigation on the nature of the type-specific antigen a. Surface washes of BS4 (agar) organisms were subjected to different chemical and enzymic treatments and examined in two-dimensional immunoelectrophoresis with rabbit antisera prepared against BS3 (agar) for disappearance or modification of the precipitin line $a$. When the surface wash was held at $\mathrm{pH}$ values between 2 and 10 at $20^{\circ} \mathrm{C}$ for 30 min and then re-adjusted to neutral $\mathrm{pH}$, there was no apparent effect on the antigen. However, the line corresponding to the antigen appeared altered and formed a distinct peak after heating the surface wash at $100{ }^{\circ} \mathrm{C}$ for 
Table 2. Immunogenicity for guinea pigs of a combination of type-specific antigen/antibody complex from BS4 (agar) organisms and killed BS4R organisms

\begin{tabular}{|c|c|c|c|}
\hline \multirow{3}{*}{$\begin{array}{l}\text { Type-specific } \\
\text { antigen/antibody } \\
\text { complex from } \\
\text { BS4 (agar) }\end{array}$} & \multirow{3}{*}{$\begin{array}{l}\text { No. of } \\
\text { c.f.u. } \\
\text { BS4R }\end{array}$} & \multirow{2}{*}{\multicolumn{2}{|c|}{$\frac{\text { No. of chambers found immune }}{\text { No. of chambers challenged }}$}} \\
\hline & & & \\
\hline & & Expt 1 & Expt 2 \\
\hline+ & - & $1 / 9$ & $1 / 9$ \\
\hline - & $10^{9}$ & $6 / 10$ & ND \\
\hline- & $10^{8}$ & ND & $1 / 9$ \\
\hline- & $10^{7}$ & ND & $0 / 7$ \\
\hline+ & $10^{9}$ & $9 / 10$ & ND \\
\hline+ & $10^{8}$ & ND & $9 / 9$ \\
\hline+ & $10^{2}$ & ND & $6 / 8$ \\
\hline- & - & $0 / 9$ & $0 / 9$ \\
\hline
\end{tabular}

ND, Not determined.

* Type-specific antigen/antibody complex was given as a course of three injections at $14 \mathrm{~d}$ intervals (the first two with Freund's complete adjuvant) of a suspension of antigen/antibody complex excised from gels; killed BS4R were given as a course of five subcutaneous injections at weekly intervals, the first at the same time as the first injection of the complex.

$\dagger$ See Table 1.

2 min at $\mathrm{pH} 7 \cdot 4$. The antigen appeared stable to treatment of the surface wash with sodium periodate $[33 \%(\mathrm{v} / \mathrm{v})$ of a $5 \%(\mathrm{w} / \mathrm{v})$ solution at $\mathrm{pH} 6.5$ and room temp. for $18 \mathrm{~h}]$ and bovine pancreatic trypsin [Sigma, type III; $0.25 \mathrm{mg} \mathrm{ml}^{-1}$ in $0.1 \mathrm{M}-\mathrm{KH}_{2} \mathrm{PO}_{4} / \mathrm{NaOH}$ buffer $\mathrm{pH} 7.5$ for $3 \mathrm{~h}$ at $37^{\circ} \mathrm{C}$ and then $18 \mathrm{~h}$ at room temp.], treatments which markedly affected other antigens in the surface wash. Interactions with pronase (BDH) under identical conditions to those for trypsin reduced the intensity of precipitin line $a$. Treatment with Triton $\mathrm{X}-100[\mathrm{BDH} ; 1 \%(\mathrm{v} / \mathrm{v})$ for $10 \mathrm{~min}$ at room temp.] prevented the formation of precipitin line $a$.

Distribution of antigens $a, b, c$ and $d$ in gonococci obtained directly from urethral pus. Originally, type-specific antigens like antigen $a$ were discovered in surface washes of three strains of gonococci that had been passaged through guinea pig chambers. Similar antigens were also found in some clones of two recent isolates of gonococci from urethral pus which had not been passaged through such chambers (Penn et al., 1978). To obtain further information on the distribution of this antigen and antigens $b, c$ and $d$ in gonococci obtained directly from human pus, five further isolates were examined. All the isolates formed some 'double highlight' colonies (Penn et al., 1977b) when first cultured on agar but, in contrast to the organisms obtained from guinea pig chambers (Penn et al., 1977b), the colonies formed rough suspensions in saline. Examination of surfaces washes of these organisms in twodimensional immunoelectrophoresis against homologous rabbit antisera showed, for four of the five isolates, the presence of lines similar to those formed by antigens $b$ and $c$. However, the surface wash of only one isolate showed a faint line similar to that formed by the typespecific antigen $a$. Cloning of the five rough isolates produced $0 / 30,0 / 30,3 / 10,3 / 12$ and $2 / 16$ smooth clones. The surface washes of organisms from these smooth clones showed lines corresponding to antigens of type $a$ (Fig. 1) in two-dimensional immunoelectrophoresis against homologous antisera. Thus, of seven recent rough isolates examined here and previously (Penn et al., 1978) five contained some organisms producing smooth suspensions in saline and antigens corresponding to the type-specific antigen $a$. However, the majority of organisms formed rough suspensions in saline and did not produce antigens of type $a$ but did produce antigens of types $b$ and $c$, which, like $a$, were not formed by surface washes of strain BSDH which had been subcultured many times since isolation. 


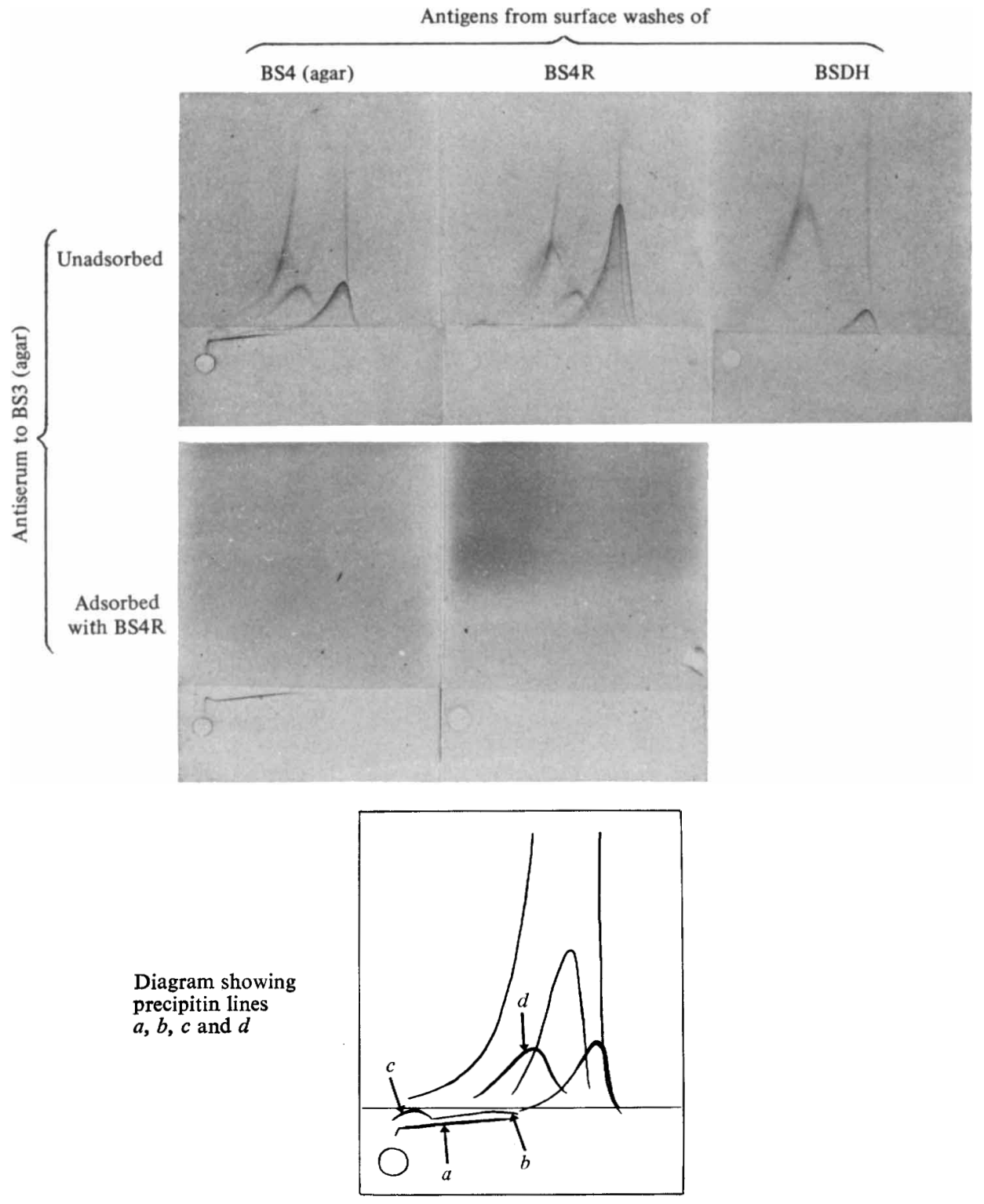

Fig. 1. Two-dimensional immunoelectrophoretic patterns of antigens in surface washes of strains BS4 (agar), BS4R, BSDH against rabbit antisera to BS3 (agar) and with the same serum after adsorption with BS4R.

\section{DISCUSSION}

The gonococci adapted to growth in guinea pig chambers and grown once on agar [BS4 (agar)] were better immunogens than organisms of the corresponding laboratory-grown strain BSDH (Table 1). These results recall similar ones using gonococci adapted to growth in mouse chambers (Arko et al., 1976). The good immunogenicity of the in vivo-adapted organisms suggested that the type-specific antigen which had been detected previously in surface washes of such organisms and not in washes of strain BSDH (Penn et al., 1978) might be an immunizing antigen. The surface washes of BS4 (agar) were immunogenic but in immunity tests (Table 2) the excised antigen/antibody complex prepared from the washes failed to immunize strongly despite a serological response to the complex. This suggested 
that additional antigens also contributed to the immunity produced by strain BS4 (agar). The guinea pigs which were immunized with the antigen/antibody complex were still susceptible to chamber infection with strain BS4, which contained about $85 \%$ smooth and $15 \%$ rough organisms, although the level of infection was lower than that in control chambers. Sometimes, smooth organisms predominated in the chambers of the immunized animals, but in about half of the chambers, rough organisms constituted the majority of the population. When such a predominantly rough population was made into a formalinkilled vaccine and inoculated (at a level at which it was inactive alone) together with the type-specific antigen/antibody complex, good protection was achieved, suggesting synergism between the two components (Table 2). It seemed that at least two antigens were involved in immunogenicity against challenge with BS4 - the type-specific antigen produced by the smooth organisms and one or more additional antigens produced by them and by the rough organisms (BS4R). The immunizing ability of BS4R when given alone at a concentration 100 -fold greater than that which was effective in combination with the antigen/antibody complex was explained by the presence in BS4R of about $4 \%$ smooth organisms which produced the type-specific antigen.

The question arises: what are the antigens present in the rough as well as the smooth organisms which contribute to immunity in addition to the type-specific antigen already recognized? The results summarized in Fig. 1 show that three prominent antigens, $b, c$ and $d$, and others less well-defined were present in surface washes of both BS4 (agar) (predominantly smooth organisms) and BS4R (predominantly rough organisms) but not in surface washes of the corresponding laboratory strain BSDH, which had not been adapted to growth in guinea pig chambers. One or more of these antigens or others not detected or absent from surface washes may protect guinea pigs in association with the type-specific antigen $a$ (Fig. 1). Antigens similar to $b$ and $c$ were present in surface washes of four out of five fresh isolates from urethral pus, all of which formed rough suspensions in saline. In agreement with previous work (Penn et al., 1978), antigens similar to the type-specific antigen $a$ could be detected in surface washes of a few smooth clones which were selected from three of the five isolates. However, rough organisms lacking antigens similar to the type-specific antigen $a$ but possessing antigens similar to $b$ and $c$ (Fig. 1) were present in greater numbers in these fresh isolates from urethral pus than smooth organisms producing antigens of type $a$ in addition to the other two. Passage through guinea pig chambers appears to select for the smooth organisms.

The type-specific antigen $a$ may be a membrane protein since it is affected by Triton $\mathrm{X}-100$ and pronase. This antigen and antigens $b, c$ and $d$ may relate to the outer membrane proteins described by Buchanan et al. (1977), Heckels \& Everson (1978) and Heckels (1978) or be connected with the capsules that have been seen on gonococci adapted to growth in guinea pig chambers (Hormaeche et al., 1978).

The observations described here underline the variability of gonococci in relation to antigenicity, immunogenicity and probably virulence. Clearly, fresh isolates from urethral pus and gonococci adapted to guinea pig chambers are mixed populations of organisms, some with more antigens than others. As regards production of immunity to infection of guinea pig chambers, at least two antigens are needed for protection against smooth organisms, one of which is not possessed by rough organisms. Most protective antigens are virulence factors (Smith, 1974) and it appears that, at least for guinea pig chambers, virulence is determined by several factors that are additive in their action, i.e. the deletion of one does not result in a complete loss of virulence but only in a reduction. Thus the smooth organisms. having a complete set of factors, seem to require the neutralization of more than one factor by the specific antibodies (or cellular immune mechanisms) for effective immunization; whereas the rough organisms, partially virulent despite loss of one or more virulence factors, may require neutralization by fewer different antibodies. Nevertheless, if antibodies have only been produced to the virulence factor not possessed by the rough organisms (i.e. 
antigen $a$ ), then the guinea pigs will not be immune to challenge by the less virulent rough organisms. The latter are normally overgrown by the more virulent smooth organisms, but here they are favoured by the selective pressure of immunity to the virulence factor which they lack. The observations on the recent isolates suggest that a similar situation - virulence factors being additive in action and mixed populations of organisms with varying complements of these factors - may exist for gonococci in humans. If this is so, a vaccine composed of a single antigenic virulence factor will not provide protection in humans against all the different members of the gonococcal population. This complication to vaccination against one strain is additional to the obvious difficulty of immunizing against a variety of immunotypes.

\section{REFERENCES}

ARKo, R. J. (1974). An immunologic model in laboratory animals for the study of Neisseria gonorrhoeae. Journal of Infectious Diseases 129, $451-455$.

Arko, R. J., Bullard, J. C. \& Duncan, W. P. (1976). Effects of laboratory maintenance on the nature of surface reactive antigens of Neisseria gonorrhoeae. British Journal of Venereal Disease 52, 316-325.

Arko, R. J. \& Wong, K. H. (1977). Comparative physical and immunological aspects of the chimpanzee and guinea pig subcutaneous chamber models of Neisseria gonorrhoeae infection. British Journal of Venereal Disease 53, 101-105.

Buchanan, T. M. \& Arko, R. J. (1977). Immunity to gonococcal infection induced by vaceination with isolated outer membranes of Neisseria gonorrhoeae. Journal of Infectious Diseases 135, 879-887.

Buchanan, T. M., Pearce, W. A., Schoolnik, G. K. \& ARKo, R. J. (1977). Protection against infection with Neisseria gonorrhoeae by immunization with outer membrane protein complex and purified pili. Journal of Infectious Diseases 136, S132-S137.

Chandler, F. W., Kraus, S. J. \& Watts, J. C. (1976). Pathological features of experimental gonococcal infection in mice and guinea pigs. Infection and Immunity 13, 909-914.

Diena, B. B., Ashton, F. E., Ryan, A. \& Wallace, R. (1978). The lipopolysaccharide (R type) as a common antigen of Neisseria gonorrhoeae. I. Immunising properties. Canadian Journal of Microbiology 24, 117-123.

HeCKels, J. E. (1978). The surface properties of Neisseria gonorrhoeae: topographical distribution of the outer membrane protein antigens. Journal of General Microbiology 108, 213-219.

HeCkels, J. E. \& Everson, J. S. (1978). The isolation of a new outer membrane protein from the parent strain of Neisseria gonorrhoeae P9. Journal of General Microbiology 106, 179-182.

Hormaeche, R. D., Thornley, M. J. \& Glauert, A. M. (1978). Demonstration by light and electron microscopy of capsules on gonococci recently grown in vivo. Journal of General Microbiology 106, 81-91.

Kwapinski, J. B. G., Cates, C., Landolfo, P., Cheng, A. \& Ronald, A. R. (1976). Immune response to a purified cytoplasmic protein of Neisseria gonorrhoeae. British Journal of Venereal Disease 52, 124-127.

Novotny, P., Broughton, E. S., Cownley, K., Hughes, M. \& Turner, W. H. (1978). Strainrelated infectivity of Neisseria gonorrhoeae for guinea pig subcutaneous chambers and the variability of the immune response in different breeds of guinea pigs. British Journal of Venereal Disease 54, 88-96.

Penn, C. W., Sen, D., Veale, D. R., Parsons, N. J., SmITH, H. \& WITt, K. (1976). Morphological, biological and antigenic properties of Neisseria gonorrhoeae adapted to growth in guinea-pig subcutaneous chambers. Journal of General Microbiology $97,35-43$.

Penn, C. W., Parsons, N. J., Sen, D., Veale, D. R. \& SMITH, H. (1977a). Immunization of guinea pigs with Neisseria gonorrhoeae: strain specificity and mechanisms of immunity. Journal of General Microbiology 100, 159-166.

Penn, C. W., Veale, D. R. \& Smith, H. (1977b). Selection from gonococci grown in vitro of a colony type with some virulence properties of organisms adapted in vivo. Journal of General Microbiology 100, 147-158.

Penn, C. W., Parsons, N. J., Veale, D. R. \& Smith, H. (1978). Correlation with different immunotypes of gonococcal antigens associated with growth in vivo. Journal of General Microbiology 105, 153-157.

RoBbins, J. B. (1977). Problems posed by gonococcal vaccines from the vantage point of a control agency. British Journal of Venereal Disease 53, 170-172.

Scales, R. W. \& Kraus, S. J. (1974). Development and passive transfer of immunity to gonococcal infection in guinea pigs. Infection and Immunity 10, 1040-1043.

SмIтH, H. (1974). Microbial interference with host defence mechanisms. Monographs in Allergy 9, 13-38.

Turner, W. H. \& Novotny, P. (1976). The inability of Neisseria gonorrhoeae pili antibodies to confer immunity in subcutaneous guinea pig chambers. Journal of General Microbiology 92, 224-228.

Veale, D. R., Smith, H., Witt, K. A., \& Marshall, R. B. (1975). Differential ability of colonial types of Neisseria gonorrhoeae to produce infection and an inflammatory response in subcutaneous perforated plastic chambers in guinea pigs and rabbits. Journal of Medical Microbiology 8, 325335.

Wong, K. H., Arko, R. J., Logan, L. C. \& Bullard, J. C. (1976). Immunological and serological diversity of Neisseria gonorrhoeae: gonococcal serotypes and their relation with immunotypes. Infection and Immunity 14, 1297-1301. 